\title{
Martian magnetism with orbiting sub-millimeter sensor: simulated retrieval system
}

\author{
Richard Larsson ${ }^{1}$, Mathias Milz ${ }^{2}$, Patrick Eriksson ${ }^{3}$, Jana Mendrok $^{3}$, Yasuko Kasai ${ }^{1}$, Stefan Alexander Buehler ${ }^{4}$, \\ Catherine Diéval $^{5}$, David Brain ${ }^{6}$, and Paul Hartogh ${ }^{7}$ \\ ${ }^{1}$ National Institute of Information and Communications Technology, Tokyo, Japan \\ ${ }^{2}$ Luleå University of Technology, Kiruna, Sweden \\ ${ }^{3}$ Chalmers University of Technology, Gothenburg, Sweden \\ ${ }^{4}$ University of Hamburg, Hamburg, Germany \\ ${ }^{5}$ Lancaster University, Lancaster, UK \\ ${ }^{6}$ University of Colorado, Boulder, USA \\ ${ }^{7}$ Max Planck Institute for Solar System Research, Göttingen, Germany \\ Correspondence to: Richard Larsson (ric.larsson@gmail.com)
}

Received: 19 April 2016 - Published in Geosci. Instrum. Method. Data Syst. Discuss.: 30 August 2016

Revised: 5 December 2016 - Accepted: 18 December 2016 - Published: 27 January 2017

\begin{abstract}
A Mars-orbiting sub-millimeter sensor can be used to retrieve the magnetic field at low altitudes over large areas of significant planetary crustal magnetism of the surface of Mars from measurements of circularly polarized radiation emitted by the $368 \mathrm{GHz}$ ground-state molecular oxygen absorption line. We design a full retrieval system for one example orbit to show the expected accuracies on the magnetic field components that one realization of such a Mars satellite mission could achieve. For one set of measurements around a tangent profile, we find that the two horizontal components of the magnetic field can be measured at about $200 \mathrm{nT}$ error with a vertical resolution of around $4 \mathrm{~km}$ from 6 up to $70 \mathrm{~km}$ in tangent altitude. The error is similar regardless of the true strength of the magnetic field, and it can be reduced by repeated measurements over the same area. The method and some of its potential pitfalls are described and discussed.
\end{abstract}

\section{Introduction}

In the past decades, there have been several proposals to fly a sub-millimeter sensor on a satellite mission to Mars. One such proposal is to fly the Far-InfraRed Experiment, presented by Kasai et al. (2012). In their work, Kasai et al. (2012) show that molecular oxygen, carbon monoxide, water (even heavy water), ozone, isotopologues of carbon dioxide, hydrogen peroxide, and various other hydrogen radicals all should have strong signals in the spectrum of Mars which they propose to observe. Additionally, wind speed parameters along the line of sight should have measurable readings. With this work we aim to develop the idea presented by Larsson et al. (2013) for remote measurement of magnetism by utilizing the Zeeman effect (Zeeman, 1897) on molecular oxygen in its ground state: $X\left({ }^{3} \Sigma_{\mathrm{g}}^{-}\right)$. It is possible to combine this work with the idea of Kasai et al. (2012) into a single instrument that is capable of measuring and mapping both meteorological parameters and crustal magnetic structures, but this work will only focus on the magnetic aspects of flying such an instrument.

The Martian magnetic field is thought to be a remnant of a past global dipole that disappeared about 3.5 billion years ago (Acuña et al., 1998). All that remains of the past dipole is several magnetic sources in the crust, which were first measured in situ by the Magnetometer-Electron Reflectometer on board the Mars Global Surveyor (MGS) orbiter (Acuña et al., 1999) down to an altitude of $100 \mathrm{~km}$. The strongest sources are located in the Southern Hemisphere, with strengths of up to $2000 \mathrm{nT}$ at $100 \mathrm{~km}$ altitude. The ongoing Mars Atmosphere and Volatile Evolution mission will provide further coverage of the magnetic field down to $\sim 150 \mathrm{~km}$ altitude in normal mode, but during week-long campaigns its periapsis will be even lower at $\sim 125 \mathrm{~km}$ al- 
titude, improving the potential to map the crustal magnetism (Jakosky et al., 2015). There are to our knowledge two planned landers, InSight (http://insight.jpl.nasa.gov) and ExoMars 2020, that will carry magnetometers to the equatorial surface of Mars.

The shape and distribution of the present field is informative for the crustal evolution of Mars (Nimmo and Tanaka, 2005). There are two main ideas about the magnetisms' formation: either a large impact demagnetized the north, leaving the south magnetized, or there was a southward migration of crustal material after the global dipole disappeared (see, e.g., Connerney et al., 2004, and Citron and Zhong, 2012, for further discussions). Regardless of the reason, the strongest crustal field should be associated with the oldest intact crustal material since these regions are linked to the times when Mars still had an effective global dipole. Identifying the reasons why the Northern Hemisphere has a lower average elevation, is younger, and also has a less magnetized crust than the Southern Hemisphere is important for questions related to, e.g., how similar Mars and Earth were in their early years. The method we propose for measuring the crustal magnetic field is useful in this regard, as it allows the determination of the magnetic field strength at different altitudes in the lower atmosphere. This means that it would help in the creation of profiles of magnetic field data that in turn can be used to estimate the depths, nature, and locations of the crustal field sources. The estimation of these parameters is beyond the scope of the paper; the interested reader may check the work by Brain et al. (2003), Connerney et al. (2004), and references therein. However, we note from Connerney et al. (2004) that there is more information about the structure of the magnetic field that is revealed at $100 \mathrm{~km}$ but is hidden at $400 \mathrm{~km}$ altitude (see their Fig. 6). If there are more structures at even lower altitudes, then these cannot be sampled by satellite magnetometers. Deep dips below $200 \mathrm{~km}$ altitude cost significant amounts of fuel due to increased air drag and therefore would shorten the lifetime of a satellite mission. Finding the crustal sources' characteristics accurately using only satellite data is thus difficult - which is why the available Martian magnetic field models differ in some regions by up to $2000 \mathrm{nT}$ (cf. Cain et al., 2003; Morschauser et al., 2014) and the range of the strongest surface field is from just above $10000 \mathrm{nT}$ up to potentially $20000 \mathrm{nT}$ (Brain et al., 2003). The characteristics of the crustal field sources strongly limit the possible processes that led to the disappearance of the past dipole and to the north-south dichotomy, making the crustal sources interesting targets for geological exploration.

\section{Method}

This work is based only on simulations. We perform limb simulations around the molecular oxygen absorption line at $368 \mathrm{GHz}$ using a measurement scenario that achieves several measurements around the same latitude-longitude tangent profile during successive satellite revolutions. These simulated measurements are fed into a retrieval toolbox that estimates the errors of the magnetic field components in the tangent profile.

Section 2 is divided as follows. The first subsection summarizes the ideas behind the approach in generic terms. The second subsection goes over the basic aspects of the modeling theory. The last subsection describes the data required for the simulations and our practical design choices. Then Sect. 3 describes and discusses the results. Finally Sect. 4 is our conclusion.

\subsection{Measurement idea}

The magnetic field influences the absorption of molecular oxygen through the Zeeman effect. Molecular oxygen exhibits the clearest Zeeman effect in the sub-millimeter region of the molecules available in the Martian atmosphere. Other molecules have either a weaker Zeeman effect or a lower volume mixing ratio and are therefore less suitable for magnetic field retrievals. The Zeeman effect changes the energy states of the molecule to split an otherwise singular absorption line into several closely separated lines as a linear function of the strength of the magnetic field. The direction of the magnetic field is important as emission and absorption are polarized by this splitting. The Martian crustal magnetic field is strong, but not strong enough to cleanly separate the split lines from the temperature and pressure broadening of the line shape. Measuring intensity peaks and valleys of the radiation on a frequency resolved grid to directly get the magnetic field strength by peak-to-peak frequency separation is not possible. Instead, the split lines act to broaden or shift the absorption profile by a few, up to hundreds of, kilohertz $(\mathrm{kHz})$. Such frequency broadening or shifting also happens from increased temperatures and from greater wind velocities.

So, an important question to ask is how we can distinguish the atmospheric effects from the magnetic effects on the absorption line. The most obvious way is to simply measure the polarization state of the radiation. Neither temperature nor wind polarizes the radiation, so the level of polarization in the split/broadened spectra is from the magnetic field in clearsky limb view. A full sampling of the polarization state of the radiation is therefore the best way to retrieve the magnetic field using the Zeeman effect. However, it is more expensive and more difficult to build a sensor capable of measuring the different polarization components (so that the components then can be combined for the total polarization state of the radiation) than it is to build a sensor capable of measuring just one polarization component. Assuming we can only measure one polarization component, is it still possible to get distinct magnetic information? Our approach has been to set up an observational strategy that observes the same limb tangent profile multiple times from several directions during a few satellite revolutions. Temperature and pressure broaden the 
absorption line shape the same regardless of observational direction through the limb. Wind shifts the frequency along just one axis. The total signal strength in a transparent passively emitting atmosphere is from the number density of the emitting molecule. What remains of the signal after these effects are accounted for is therefore the polarization state and frequency shift due to the Zeeman effect.

\subsection{Theoretical considerations}

We use the Atmospheric Radiative Transfer Simulator (ARTS; Buehler et al., 2005; Eriksson et al., 2011) to simulate measurements. ARTS is a multi-purpose radiative transfer simulator, originally developed for limb sounding of Earth's atmosphere in the microwave spectral range. It currently handles microwave to infrared radiation and can simulate the full Stokes vector in arbitrary viewing geometry, with or without scattering. Applications for Earth's atmosphere have been as diverse as simulating detailed millimeter-wave three-dimensional radiative transfer through clouds (Davis et al., 2007) and simulating the infrared outgoing long-wave radiation leaving Earth to space across the full spectrum (Buehler et al., 2006). Recently, ARTS was adapted to handle other solar system planets, such as Mars. The retrieval toolbox Q-package (Qpack; Eriksson et al., 2005) is used to determine the magnetic field component error. Together, these code bases set up our retrieval system. The source codes of both software packages are freely available under the General Public License (GNU) at http://www.radiativetransfer.org.

The approach to radiative transfer taken by ARTS is to calculate the monochromatic pencil-beam polarized radiative transfer equation in Stokes formalism along the path of the radiation through a three-dimensional inhomogeneous atmosphere and magnetic field. Antenna size, sensor characteristics, and polychromatic signal averaging are considered (as described by Eriksson et al., 2006). The Zeeman effect module of ARTS (Larsson et al., 2014) is applied for these calculations to simulate the left circular polarization component as observed by the simulated sensor for a $20 \mathrm{MHz}$ passband of 201 channels of $100 \mathrm{kHz}$ Gaussian shape surrounding the central absorption line at $368 \mathrm{GHz}$. The ARTS Zeeman effect module has been validated using ground-based measurements (Navas-Guzmán et al., 2015) and by comparisons with meteorological satellite measurements (Larsson et al., 2016), in applications considering the radiation in different states of polarization. Circular polarization is noticeably more influenced by the magnetic field than linear polarization, and $10 \mathrm{MHz}$ on both sides of the line captures most of the information given by the Zeeman effect. For circular polarization, the magnetic signal is strongest when the local magnetic field vector points directly at or is pointing directly away from the sensor (along the track of the measured radiation). So in limb-viewing geometry radiative transfer, only the horizontal magnetic field components are important at the tangent point. We expect some sensitivity to the radial compo- nent at higher altitudes than the tangent altitude (since part of the radial component is then along the line of sight), but the sensitivity to the radial component is expected to be low since most of the signal is from around the tangent point. If we measure linear instead of circular polarization, then these measurements are sensitive to both the radial component and the horizontal components of the magnetic field. However, the magnetic signal is significantly weaker for linear than for circular polarization, so the retrieved magnetic field would be noisier. The ARTS simulations also give the Jacobian matrix for specified retrieval quantities (in our case the magnetic field components and, for testing, temperature, wind components, and the molecular oxygen volume mixing ratio).

We use a moderately nonlinear error characterization method, as presented by Rodgers (2000), to estimate the errors associated with a simulated measurement on a retrieval quantity. This error characterization is from

$$
\begin{gathered}
\boldsymbol{x}=\boldsymbol{x}_{\mathrm{a}}+\mathbf{S}_{\mathrm{a}} \mathbf{K}^{\top}\left(\mathbf{K S}_{\mathrm{a}} \mathbf{K}^{\top}+\mathbf{S}_{\epsilon}\right)^{-1} \\
{\left[\boldsymbol{y}-\mathbf{F}(\boldsymbol{x})+\mathbf{K}\left(\boldsymbol{x}-\boldsymbol{x}_{\mathrm{a}}\right)\right],}
\end{gathered}
$$

where $\boldsymbol{x}$ is the derived atmospheric variables and magnetic field, $\boldsymbol{x}_{\mathrm{a}}$ is the a priori atmospheric variables and magnetic input at different retrieval grid points (atmospheric pressure level, latitude, longitude), $\mathbf{S}_{\mathrm{a}}$ is the covariance matrix of the a priori, $\mathbf{K}$ is the Jacobian matrix, $\mathbf{S}_{\epsilon}$ is the covariance matrix of the instrument error, $\boldsymbol{y}$ is the simulated measurement vector (made up of several individual measurements), and $\mathbf{F}(\boldsymbol{x})$ is the forward-model simulations. With measurements $\mathbf{F}(\boldsymbol{x}) \neq \boldsymbol{y}$, however, with pure simulations the terms are identical. Therefore, the error itself is found from $\mathbf{F}(\boldsymbol{x})=\boldsymbol{y}-\boldsymbol{\epsilon}$, where $\epsilon$ is the random noise of the observation - this "noise" encompasses every error not accounted for by the simulated measurement, especially defined instrumental errors. Thus the retrieval error of Eq. (1) is $\mathbf{S}_{\mathrm{a}} \mathbf{K}^{\top}\left(\mathbf{K S}_{\mathrm{a}} \mathbf{K}^{\top}+\mathbf{S}_{\epsilon}\right)^{-1} \boldsymbol{\epsilon}$. We also estimate the smoothing of the calculations by the matrix $\mathbf{S}_{\mathrm{a}} \mathbf{K}^{\top}\left(\mathbf{K S}_{\mathrm{a}} \mathbf{K}^{\top}+\mathbf{S}_{\epsilon}\right)^{-1} \mathbf{K}$, which is called the averaging kernel. This matrix gives information on the measurement response of the system and the vertical resolution of the measurements. The method above gives a linear error estimate; even if a problem is inherently nonlinear, the error is often still linear (Rodgers, 2000).

About the retrieval grid, as explained in the previous subsection, several measurements of radiation from the same tangent profile can be combined to find the magnetic field components. The observational geometry is important as the individual measurements will observe different parts of the atmosphere at higher altitudes but the same parts of the atmosphere at lower altitudes. We therefore use a retrieval grid that takes the three-dimensional inhomogeneous atmosphere and magnetic field into account by setting a 3-by-3 grid of latitudes and longitudes, with the central grid point at the latitude and longitude of the tangent profile. The latitude grid positions are separated by a change of $2^{\circ}$, with the same hor- 
izontal distance separating the longitude grid positions. We use a vertical retrieval grid separation of $2 \mathrm{~km}$ from 0 up to $100 \mathrm{~km}$. So $\boldsymbol{x}_{\mathrm{a}}$ is a $3 \times 3 \times 51$ long vector of inputs per retrieval quantity. We note that the measured magnetic field for the altitude range we have considered (below $100 \mathrm{~km}$ ) is essentially the planetary crustal magnetism (Brain et al., 2003). External fields (interplanetary magnetic fields draping around the conductive ionosphere and induced ionospheric magnetic fields) play a role at higher altitude, where the solar wind interacts with the upper atmosphere. In areas of significant crustal magnetism, the influence of external fields starts at altitudes around $500 \mathrm{~km}$ (Fig. 11 of Brain et al., 2003); therefore they are not a concern here.

For $\mathbf{S}_{\mathrm{a}}$, we assume that there is no correlation between the different retrieval quantities but that there is some correlation in spatial distance. For the tests we have performed on the wind, temperature, and volume mixing ratio retrievals, we use covariance matrices that work for Earth. For the magnetic field components, since the crustal magnetic field is a mostly static variable at the altitudes below $100 \mathrm{~km}$ where we simulate the measurements, its covariance matrix should also be mostly static. However, in a Bayesian framework, the covariance matrix should describe the knowledge we have of the magnetic field at the time of measurement. As mentioned earlier, the strongest difference between the models compared in Morschauser et al. (2014) was $2000 \mathrm{nT}$, but many sources differ less. So different models give different results. For simplicity we set the uncertainty of the magnetic field to $1000 \mathrm{nT}$ at all altitudes, and the correlation in altitude is $e^{-1}$ after 1 order of magnitude of atmospheric pressure change. We also set a small correlation between horizontal grids of $e^{-1}$ after a horizontal distance equivalent to $1.5^{\circ}$ of latitude change.

\subsection{Model inputs}

\subsubsection{Orbit and sensor considerations}

A circular orbit at $330 \mathrm{~km}$ altitude with around a $2 \mathrm{~h}$ period, $97^{\circ}$ inclination, and ascending node at $0^{\circ}$ is used in all simulations. This orbit was selected ad hoc, such that it is able to cover Mars in a short time frame with measurements covering almost a full circle in azimuthal resolution. Other orbital parameters can be used to retrieve the magnetic field as well but require different considerations. An elliptical orbit, for instance, will have different vertical resolutions depending on true anomaly, and a less inclined orbit means that a different measurement scheme has to be adopted and that fewer poleward latitudes can be sounded - most likely with increased measurement noise. With enough measurements the measurement noise could be reduced regardless of orbit. However, the quantitative details of the change of the measurement noise to any specific orbit change have to be worked out on a per-orbit basis, so how much the noise can be reduced is not possible to tell without doing specific simulations for a given set of orbital parameters.

With this choice of orbital parameters, we model a sensor with a $30 \mathrm{~cm}$ diameter antenna for a vertical resolution of about $4 \mathrm{~km}$ at the tangent points sampled in limb sounding by an orbiter at $330 \mathrm{~km}$ altitude. The antenna size was chosen for these simulations because it is reasonably small. We will only simulate left circular polarized radiation, and we assume $1500 \mathrm{~K}$ single-sideband system noise temperature. This number is a rough extrapolation to lower frequencies from the expected system noise temperature of the Jupiter Icy Moons' Sub-millimeter Wave Instrument's $600 \mathrm{GHz}$ band (see Sobis et al., 2011, 2014, and Treuttel et al., 2016, for more details on the JUICE/SWI receiver). This extrapolation simply scales the SWI number by the frequency ratio of the channels and rounds the results up for good measure. The engineering has to be done to give a more exact number. We enforce $2 \mathrm{~s}$ as the time of a measurement, dedicating one-third to calibration efforts and the remaining $\sim 1.3 \mathrm{~s}$ as integration time.

The position of the tangent profiles tracks this orbit as a pseudo-orbit with the same orbital parameters but with an ascending node offset by half the longitude drift of a full satellite revolution. These tangent profiles can be observed in limb geometry four times within about two revolutions: for each revolution, once before passing the tangent profile and once after passing the tangent profile. The tangent altitudes we select to observe this tangent profile extend from 6 to $78 \mathrm{~km}$ altitude, with a $3 \mathrm{~km}$ vertical separation for 25 tangent altitudes. So a total of 100 individual measurements are considered ( 25 measurements $\times 4$ observation sets) in the error characterization. We call the grouping of all these individual measurements for a given tangent profile a measurement block. The error characterization of this work is from these measurement blocks. The results in the next sections track the tangent profile pseudo-orbit through seven complete revolutions at a distance of $15^{\circ}$ of true anomaly between two retrieval profiles. This is a sufficient number of revolutions so that we can give the global error characterization.

An example of observation geometry and orbit can be seen in Fig. 1 (the Supplement provides additional details). As mentioned, we simulate 201 channels for the sensor. With 100 individual measurements in a measurement block, $\boldsymbol{y}$ is a 20100 long vector, and $\mathbf{K}$ is a $20100 \times(3 \times 3 \times 51 \times n)$ large matrix, where $n$ is the number of retrieval quantities (usually three for the magnetic field components) and the other numbers are from the retrieval grid. The position of all 100 of these individual measurements in the example of Fig. 1 is shown in panel $a$. Panel $b$ of the same figure shows the grouping of individual measurements for one set of observations of the tangent profile. We call this smaller grouping a measurement cluster. A satellite moving towards a tangent profile will, by geometrical necessity, see the tangent altitude increase the closer it gets to the profile, and vice verse a satellite moving away will see the tangent altitude decrease the farther away it gets. Finally, panel c shows that there is a 

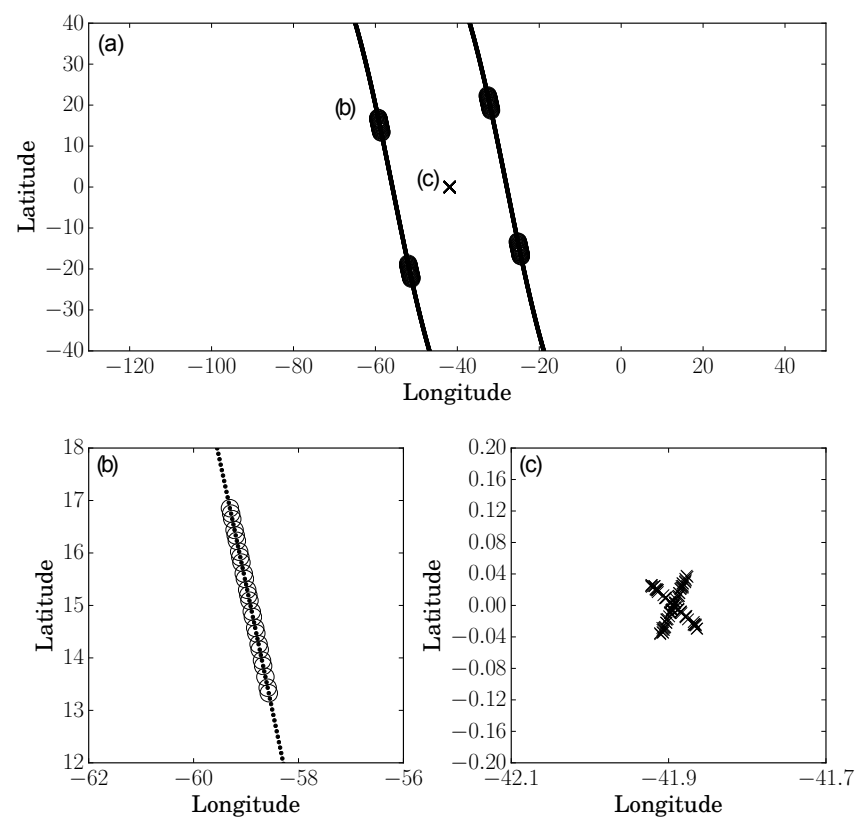

Figure 1. Example of the geometry of a measurement block. Panel (a) shows the orbit of a satellite as two thin lines indicating two satellite revolutions: the satellite positions during the two observation sets during each revolution in the measurement block as larger circles, and the mean tangent position of the measurement block as a cross. This example retrieval point is above a location of moderate modeled magnetism strength near the equator (750 nT at surface altitudes crustal magnetism model by Cain et al., 2003). Panel (b) zooms in to show the satellite positions at one of the clusters of satellite positions. Panel (c) zooms in to show the tangent positions. The original intent of the measurement block was to observe in the center of this block of individual measurements.

slight drift in tangent point positions between different measurements. It is thus expected that the tangent position will drift slightly; the greater circle of positions that can observe a tangent altitude does not strictly intersect the satellite orbit at discrete time intervals. The drift is accounted for in the calculations of $\mathbf{K}$ and $\boldsymbol{y}$.

The only spectroscopic effect included in our radiative transfer calculations is the contribution of the $368 \mathrm{GHz}$ molecular oxygen absorption line. We select this absorption line because the Zeeman effect line splitting frequency is linear in magnetic field strength. So the lower the frequency, the stronger the signal from the magnetic field becomes as Doppler broadening is also linear with absorption line frequency. The reason we are not simulating one of the $60 \mathrm{GHz}$ molecular oxygen band lines is that we consider the antenna diameter required to have a decent vertical resolution in limb view as too large to reasonably fly the sensor. Our line data are from a recently compiled planetary toolbox, which gives line pressure broadening as a function of atmospheric composition for mixtures of common planetary species ${ }^{1}$ by Mendrok and Eriksson (2014), who assume that the molecular oxygen pressure broadening by carbon dioxide is $\sim 20 \mathrm{kHz} \mathrm{Pa}^{-1}$ at $296 \mathrm{~K}$ for our absorption line. This is directly taken from the $118 \mathrm{GHz}$ line value presented by Golubiatnikov et al. (2003). This spectroscopic toolbox allows for direct calculations in Mars' carbon-dioxide-rich atmosphere. Note that the collision-induced absorption between pairs of carbon dioxide molecules is also important for the total absorption in our frequency range. Using the model by Ho et al. (1971), we estimate that it can be as important as adding a $20 \mathrm{~K}$ baseline signal for a pencil beam tangential to the surface. This added absorption is not sufficient to make the atmosphere opaque. We can anyways ignore this effect because at $6 \mathrm{~km}$ altitude - our lowest tangent altitude - the collision-induced absorption is much weaker than at the surface. Still, we note this as a lacking feature to be added in the future.

To summarize the orbit and sensor constraints, we present Fig. 2 to give an overview of how a simulation of a measurement block looks with the assumptions outlined above. Panel a contains peak brightness temperature measurements for two clusters (looking ahead and behind the tangent point) for one revolution, and again measurements for two clusters for another revolution, with each cluster having 25 measurements at tangent altitudes between 6 and $78 \mathrm{~km}$. As expected, the main contribution to the signal strength is the tangent altitude (through which the column number densities are regulated). At $6 \mathrm{~km}$ measurement tangent altitude, the radiation signal has a peak brightness temperature of about $140 \mathrm{~K}$, and at $78 \mathrm{~km}$ the same number is about $10 \mathrm{~K}$. The sinusoidal pattern of measurements in each cluster comes from the resolution of the spectra: the peaks are at the line centers, and the valleys are away from the line centers. Only a very small part of this signal is influenced by the magnetic field. This part of the signal is not clear from the brightness temperature in panels $a-b$ but is instead shown in the Jacobian panel c. We see that the magnetic signal is of the order of about $0.2 \mathrm{mK} \mathrm{nT}^{-1}$ as strongest per $2 \mathrm{~km}$ altitude level per $100 \mathrm{kHz}$ spectrometer channel bin. The resolution of the antenna is indicated from the coverage of the circular central disk of the Jacobian panel. Again, the simulated full-width vertical resolution is around $4 \mathrm{~km}$ for a $30 \mathrm{~cm}$ antenna. The covariance matrix is shown for completeness in panel d. The figure shows neither the full $\mathbf{K}$ nor the full $\mathbf{S}_{\mathrm{a}}$ matrices but is zoomed in on a single sub-matrix. Nevertheless, the figure presents the basic retrieval setup. The only missing entry required for the error characterization is $\mathbf{S}_{\epsilon}$. This matrix is not shown because it is simply filled with a constant describing the noise of the sensor. This constant is the square root of the system noise equivalent temperature divided by the integration time times

\footnotetext{
${ }^{1}$ Our atmospheric and spectroscopic data are available in Extensible Markup Language files designed for ARTS via http://www. radiativetransfer.org.
} 

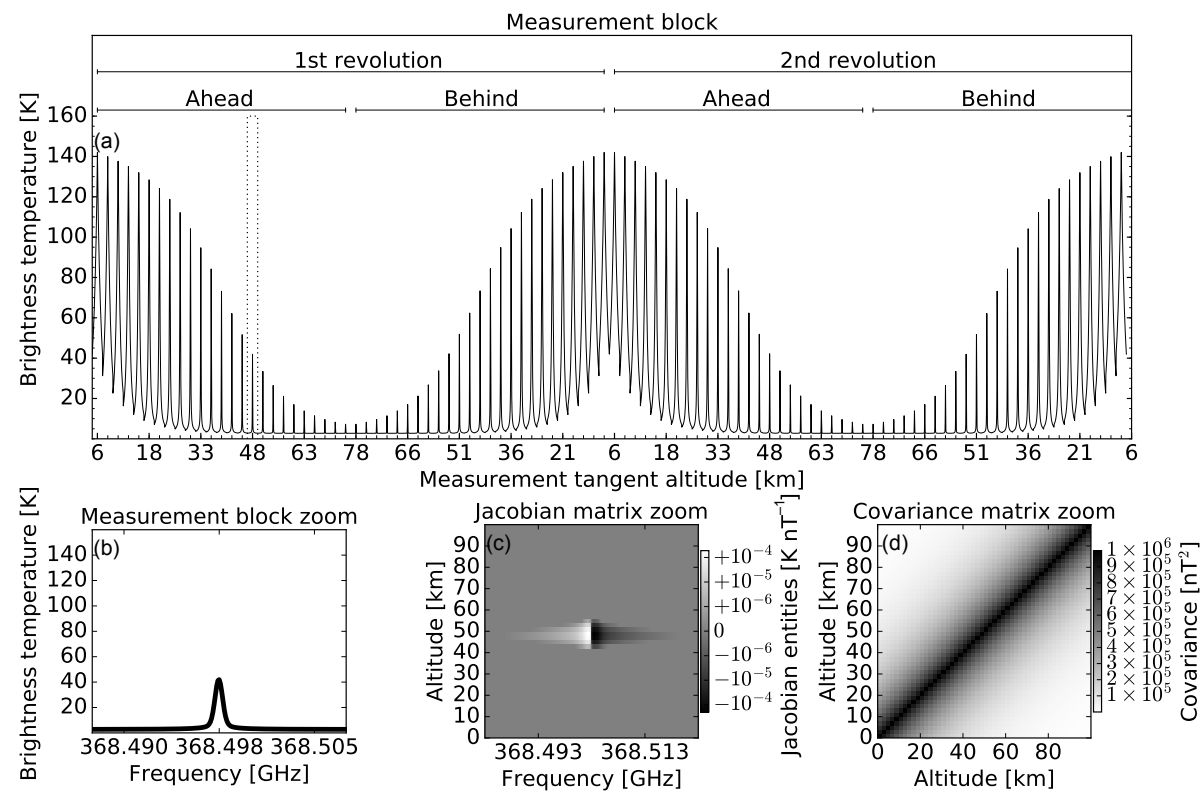

Figure 2. Example retrieval scenario. This is $\boldsymbol{y}$, part of $\mathbf{K}$, and part of $\mathbf{S}_{\mathrm{a}}$ from the example in Fig. 1. Panel (a) is the simulated measurements $\boldsymbol{y}$ of ARTS with individual clusters marked by their revolution number and looking direction. Each peak corresponds to an individual measurement - the horizontal axis gives the tangent altitudes. The bottom row zooms in on the marked region of $\boldsymbol{y}$ to show an individual simulation at $48 \mathrm{~km}$ tangent altitude (b), a zoom on the transpose of the $\mathbf{K}$ sub-matrix at the tangent point latitude-longitude grid point for one magnetic component (c), and its corresponding $\mathbf{S}_{\mathrm{a}}$ sub-matrix (d).

the spectrometer channel full width - which turns out to be just below $0.1 \mathrm{~K}^{2}$.

\subsubsection{Atmosphere and magnetic field models}

The molecular oxygen profile is assumed constant at a volume mixing ratio of 1400 parts per million volume, which follows the profile reported by Hartogh et al. (2010b). This profile was derived by observations of the Heterodyne Instrument for the Far Infrared (HIFI) on Herschel as part of the Herschel Solar System Observations program (Hartogh et al., 2009) by using a temperature profile derived at the same day from carbon monoxide observations (Hartogh et al., 2010a). At altitudes above $90 \mathrm{~km}$, this constant mixing ratio is not valid (as shown by measurements presented by Sandel et al., 2015), and it is likely not valid at our lower altitudes either. However, since we can see the molecular oxygen radiometric signal even at $78 \mathrm{~km}$ altitude, and since a changed mixing ratio only affects the total signal strength, the magnetic signal - that is, the relative polarization and splitting caused by the magnetic field - is not affected by this different mixing other than lowering or increasing the sensitive altitude range. With this selection of volume mixing ratio, we are not sensitive to molecular oxygen at altitudes much higher than $78 \mathrm{~km}$. This is seen by the low signal strength at this altitude in Fig. 2a and the reduction compared to lower tangent altitudes. Additionally, panel $\mathrm{c}$ of the same figure shows that most of the signal is from around the tangent point, which is a property of limb observation geometry. Higher-altitude molecular oxygen content is therefore not important for our results.

We use the northern spring (solar longitude $L_{\mathrm{s}}=0^{\circ}$ ) carbon dioxide volume mixing ratio, temperature profile, wind profile, and pressure profiles from the ARTS planetary toolbox by Mendrok and Eriksson (2014), who base it on the Laboratoire de Météorologie Dynamique's global circulation model (LMD GCM) by Forget et al. (1999). These atmospheric profiles only provide global averages during the season. We thus ignore most effects of time since we are interested in demonstrating the feasibility of the technique of magnetic field measurements on a general basis. Presented errors are hence of average character. The a priori atmospheric profiles can be constrained by the measurements, but we have not taken this into account in any of our simulations shown in this work, though we give an example of what adding the atmospheric components to the error characterization means for the errors of the magnetic components. If the atmospheric parameters are not stable over the $2 \mathrm{~h}$ of a revolution, then the evolution of the temperature and wind profiles must be accounted for in the retrieval setup. This is still possible without changing the theoretical formalism of the retrieval setup if the problem remains moderately nonlinear, but it complicates the preparation of the atmospheric data and the simulations. From a theoretical point of view, the error characteristics of the magnetic field will remain moderately nonlinear even if the temperature, wind, and volume mixing ratio are accounted for in the retrieval problem because there 

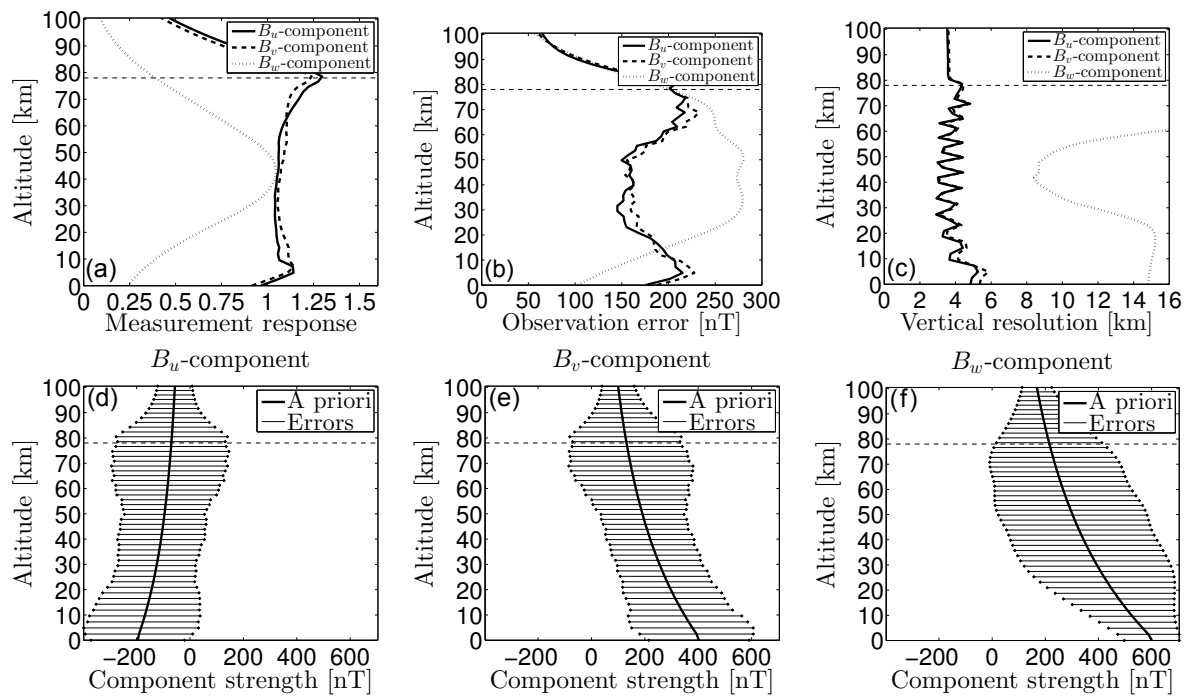

Figure 3. Retrieval information for Fig. 1. The first row shows Qpack output following Eq. (1). Panel (a) shows the response function per magnetic field component, (b) shows the observation error per magnetic field component, and (c) shows the vertical resolution per magnetic field component (zigzag is from discrete number of vertical limb measurements at $3 \mathrm{~km}$ spacing). The lower row (d-f) compares this observational error to a priori magnetic field components. The horizontal dashed line shows the highest tangent altitude.

should be no correlation between the atmospheric parameters and the magnetic field.

The primary magnetic field in our altitude range is the crustal magnetic field (Brain et al., 2003). Our model magnetic field is from the spherical harmonics fit to a selection of Mars Global Surveyor crustal magnetic field vector data by Cain et al. (2003). The magnetic field in ARTS is an extraction of the fit of Cain et al. (2003) that has been gridded at a global resolution of $0.5^{\circ}$ latitude, $0.5^{\circ}$ longitude, and $5 \mathrm{~km}$ altitude from the surface up to $100 \mathrm{~km}$ altitude. This means that the magnetic field is allowed to change along the path of the radiative transfer in our simulations. If the magnetic field had changed more dramatically through the radiative transfer than it does, then this might have reduced the magnetic signal by changing the polarization of the signal propagation. Since this is not a problem that we encounter, we will not pursue any ideas on how to deal with it. The model by Cain et al. (2003) is based on a 90th-order Legendre polynomial, so the spatial resolution is limited to $\sim 130 \mathrm{~km}$. As mentioned, other models, such as that of Morschauser et al. (2014), differ from that of Cain et al. (2003) by about $2000 \mathrm{nT}$ at most (see Fig. 9 of Morschauser et al., 2014) but are otherwise close. This $2000 \mathrm{nT}$ difference is therefore an estimation of the accuracy of the magnetic field components with the present data. Some authors (Brain et al., 2003) speculate that the strongest field strength at the surface is up to $20000 \mathrm{nT}$. Cain et al. (2003) give the strongest field as $12000 \mathrm{nT}$, so $8000 \mathrm{nT}$ serves as an estimate of the potentially largest discrepancy today of the crustal magnetic field strength of Mars.

\section{Results and discussions}

The structure of this section is that we begin by presenting the results of running the measurement block of Fig. 1. This example is used as a generic measurement block, and noise levels are lower or higher depending on the geometry of observations. We then present an average for many observations spread out globally. There is a final summary at the end of this section showcasing where our results suggest that the proposed measurements would be useful given the expected noise levels of individual measurement blocks. Note that we define the magnetic field components as follows: $B_{u}$ is the east-west component of the magnetic field, $B_{v}$ is the northsouth component, and $B_{w}$ is the radial component.

\subsection{Example measurement}

Figure 3 shows the results of running the retrieval system for the tangent profile example of the measurement block in Fig. 1 with the simulated measurements and Jacobian as in Fig. 2. It shows that the measurement response is good (i.e., around unity) for the $B_{u}$ and $B_{v}$ components (horizontal components) from the ground up to about $70 \mathrm{~km}$ for 16 mostly independent measurements of the magnetic field in the tangent profile. The $B_{w}$ component (vertical component) has a poorer response than the other two components, but the measurements are in this case sensitive to $B_{w}$ at altitudes around $40 \mathrm{~km}$. The vertical resolution is about $4 \mathrm{~km}$ for $B_{u}$ and $B_{v}$ over the entire sensitive altitude range because it is perpendicular to the line of sight in the limb geometry, which reflects the achievable resolution for the simulated antenna size and tangent profile altitude spacing. The vertical reso- 
lution is much worse for $B_{w}$ between 8 and $15 \mathrm{~km}$. The observational error that $B_{u}$ and $B_{v}$ experience varies between around 150 to $200 \mathrm{nT}$ in the sensitive altitude range. The observational error that $B_{w}$ experiences is much worse, but it settles close to $300 \mathrm{nT}$ at sensitive altitudes. Note that the observation errors are low where the measurement response is low; without measurements there are no errors due to observations.

As for vertical structures, the main limitation at lower altitudes is that pressure broadening hides the magnetic signal, and the main limitations at higher altitudes is that there is very little molecular oxygen due to the lower pressure. The optimal magnetic signal is from around $40 \mathrm{~km}$, which is why the noise at these altitudes is lower (panel $b$ ).

$B_{w}$ is more difficult to detect than the other two components because of the characteristics of the Zeeman effect. The multiple measurements from different azimuthal directions allow the angle of the magnetic field in the horizontal plane to be tested almost directly, but $B_{w}$ acts only to broaden the absorption line in limb view. It is therefore difficult to measure it without more constraints. In fact, if we add retrievals of wind, temperature and molecular oxygen volume mixing ratio to the retrieval problem - thus reducing the constraints on the retrieval problem - the measurement response to $B_{w}$ is no longer good at any altitude. So we will ignore $B_{w}$ from here on since it is not measurable by the presented method. The horizontal magnetic components are not affected by the lessened constraints on the atmospheric parameters.

\subsection{Suggested operational setup}

The average observation errors expected for our suggested operational setup scenario are presented in Fig. 4. We simulate only seven revolutions, or about half a Martian day, of measurements, since this gives a good coverage for the whole Martian globe with our ad hoc orbit.

We find that the global average profile observation error for the $B_{u}$ component is $170 \mathrm{nT}$ and that the same average observation error for the $B_{v}$ component is $200 \mathrm{nT}$. Near the equator, the two components have similar observation errors (almost identical to what is shown in Fig. 3). Closer to the poles, $B_{u}$ has better observation errors, and $B_{v}$ has worse observation errors. The reason for this is the azimuthal angles of the observation geometry of a measurement block. With the selected orbit and observational strategy, the tangent profiles are observed from the northeast/west and southeast/west azimuthal angles at the equator. Near the poles, however, the azimuthal angles are almost aligned with the east-west direction. On one hand, this indicates that it is possible to improve $B_{v}$ observation errors at polar latitudes to the same levels as $B_{u}$ by more observations from directly north or south of the tangent profiles. Similarly, it also indicates that it is possible to improve the equatorial observation errors for both horizontal components to the levels of $B_{u}$ at polar latitudes by focusing on east-west or north-south observation geometry

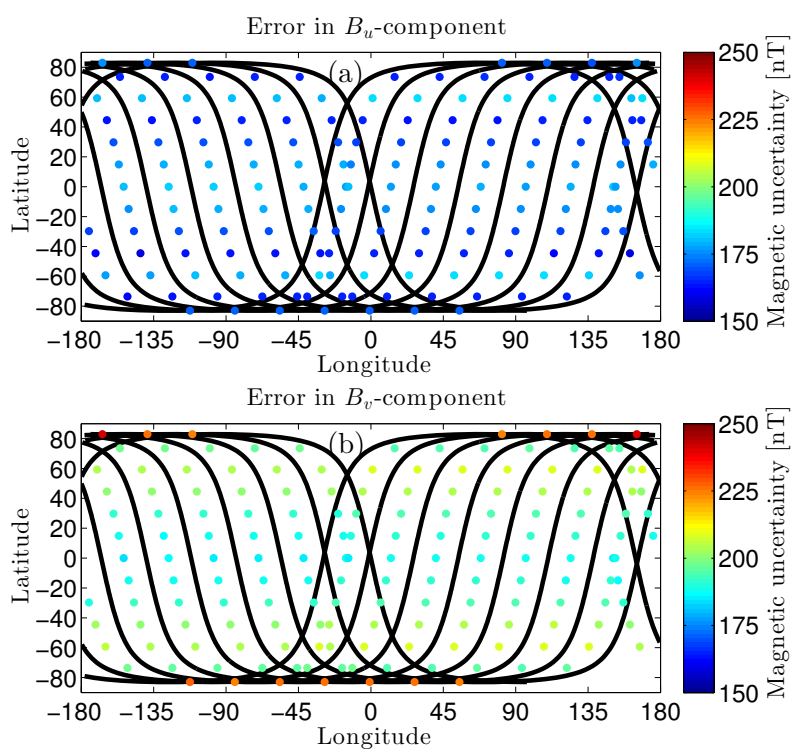

Figure 4. Suggested operational setup scenario. The black lines represent the orbit through seven revolutions. The maps show the estimations for errors per component averaged between 6 and $78 \mathrm{~km}$ altitude.

depending on the component of interest. On the other hand, if the inclination of the orbit is such that there will be fewer measurements at higher latitudes, then the errors in the $B_{v}$ component will be worse than shown in Fig. 4. Clearly, a highly inclined orbit is beneficial for the suggested measurements when compared to a less inclined orbit.

\subsection{Simple estimation of measurable area and a comparison to satellite magnetometer}

We prepared Fig. 5 to show the areas over the Martian surface where these measurements would be useful. This plot shows the modeled magnetic field strength extracted at different altitudes from Cain et al. (2003). At $40 \mathrm{~km}$ altitude, an area covering about $36 \%$ of Mars total area has magnetic field strengths above $200 \mathrm{nT}$, with the southern sources being stronger than the northern sources. The areas with more than $1000 \mathrm{nT}$ field strength cover about $4 \%$ of the surface at this altitude. So our suggested measurements are estimated to provide information on the Martian magnetic field over somewhere between 4 and $36 \%$ of the area of Mars at $40 \mathrm{~km}$ altitude, i.e., in areas where the crustal magnetism is significant. The same area percentages at $20 \mathrm{~km}$ are $9 \%$ above $1000 \mathrm{nT}$ and $49 \%$ above $200 \mathrm{nT}$, and at $60 \mathrm{~km}$ the numbers are 2 and $27 \%$, respectively. For the rest of the area of Mars, the method is only capable of confirming the sub-sensitivity surface field strengths of the Cain et al. (2003) model.

Comparing the $200 \mathrm{nT}$ per $4 \mathrm{~km}$ noise attainable by submillimeter measurements to satellite-borne magnetometer measurements by Mars Global Surveyor offers some additional information into the limitations and possibilities of the 

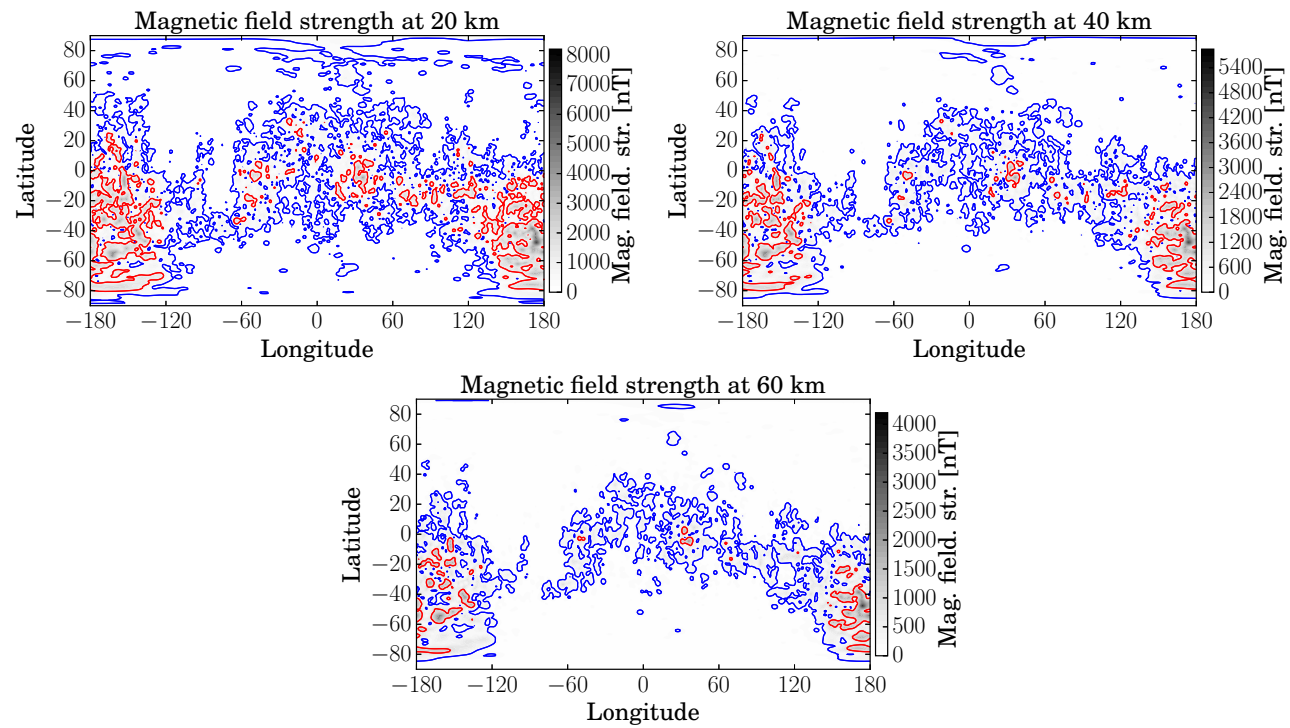

Figure 5. The modeled magnetic field strength of Mars at 20, 40, and $60 \mathrm{~km}$ altitude from Cain et al. (2003). The red contour marks $1000 \mathrm{nT}$, and the blue contour marks $200 \mathrm{nT}$.

sub-millimeter measurements. The MGS magnetometer had noise levels of only $3 \mathrm{nT}$ during its deep dips to $\sim 100 \mathrm{~km}$ (Acuña et al., 1998). At $100 \mathrm{~km}$, almost $99 \%$ of the area of Mars has a magnetic field strength stronger than $3 \mathrm{nT}$, with $76 \%$ of the area having a magnetic field strength over $15 \mathrm{nT}$ (Cain et al., 2003). How much area a satellite-borne magnetometer can cover at $100 \mathrm{~km}$ altitude during a mission is therefore mostly limited by the amount of fuel brought with the satellite to maintain a good orbit after the deep dips. Measurements far above $100 \mathrm{~km}$ altitude contribute less to understanding the structures of the magnetic field necessary to estimate the strengths, shapes, and locations of the crustal sources. This is because the structures of the magnetic field are hidden by the added distance (as seen in, e.g., Fig. 6 by Connerney et al., 2004). The higher altitude of these more stable orbits are thus less interesting in regards to crustal sources but nevertheless offer much better noise levels. The noisier sub-millimeter measurements are able to sense lower altitudes, and, in contrast to the magnetometer, this can be done from a stable orbit. We consider that the measurements of the satellite-borne magnetometers and the sub-millimeter sensors are complementary to one another, with satellite magnetometers able to more strictly limit the orientation and strength of the field and sub-millimeter spectrometers more capable to sample low-altitude structures.

Finally, we remind the reader that the magnetic field strength is mostly static and will smoothly decrease in magnitude with increasing altitude. These potentials have not been used in the retrieval system presented in this work. Starting with the altitude, we find 16 data points of errors of about $200 \mathrm{nT}$ per component from $6 \mathrm{~km}$ altitude up to $70 \mathrm{~km}$ altitude. Using these to fit to a model of smoothly decreas- ing magnetic field magnitude, the noise should reduce by the square root of the number of independent data points, potentially fitting such a model to an error of about $50 \mathrm{nT}$ per tangent profile. As also mentioned, we only consider measurements taking place during less than $24 \mathrm{~h}$ in this paper. Reasonably, a mission with a stable orbit can survive substantially longer. Throughout the lifetime of the entire mission it will be possible to measure the static magnetic field over the same areas multiple times. The error of $200 \mathrm{nT}$ per $4 \mathrm{~km}$ per horizontal component is thus just a beginning and can be reduced continuously during the mission via proper postprocessing techniques. It is beyond the scope of this work to analyze such post-processing in detail. We will leave such details to the future, if or when the sensor concept described in this work flies to Mars.

\section{Conclusions}

We have described an idea that makes measurements of the Martian near-surface crustal magnetic field profiles possible using remote-sensing techniques by combining several limb observations. We have performed radiative transfer simulations for radiation measurements from the atmosphere of Mars for one orbiting sensor. These radiative transfer simulations have been fed into a retrieval toolbox to find sensitivities to the magnetic field. Our work shows that limb observations of the sub-millimeter radiation of one absorption line of molecular oxygen can be used to measure two of the three components (the horizontal components) of the magnetic field with around $200 \mathrm{nT}$ accuracy between 6 and $70 \mathrm{~km}$ altitude. The vertical resolution of such measurements will be about $4 \mathrm{~km}$ for a $30 \mathrm{~cm}$ diameter antenna measur- 
ing the $368 \mathrm{GHz}$ line from an orbit at $330 \mathrm{~km}$ altitude. The described measurements are sensitive to the magnetic field strength over about one-third of the Martian surface, which is the area where significant localized planetary magnetic fields exist. We have made few assumptions on correlations in the retrieval system to let our method enhance measurement sensitivities rather than a priori sensitivities. With reasonable assumptions on the magnetic source fields or with different orbit/observation geometry, it should be possible to reduce the noise further with proper post-processing techniques. We suggest flying a sensor capable of measuring the molecular oxygen absorption line at $368 \mathrm{GHz}$ on a satellite mission to Mars to make a detailed map of the Martian crustal magnetic field and thereby help - in combination with available satellite magnetometer data - to find the depths, shapes, and positions of the crustal field sources.

\section{Data availability}

The data and the software are linked and described in the text. Please refer to http://www.radiativetransfer.org for both data and models. ARTS is the main focus there. The Qpack model is part of atmlab, and the atmospheric data are part of arts-xml-data.

\section{The Supplement related to this article is available online at doi:10.5194/gi-6-27-2017-supplement.}

Acknowledgements. We want to acknowledge all those in the ARTS user community who have contributed to the ARTS development.

Edited by: L. Vazquez

Reviewed by: three anonymous referees

\section{References}

Acuña, M. H., Connerney, J. E. P., Wasilewski, P., Lin, R. P., Anderson, K. A., Carlson, C. W., McFadden, J., Curtis, D. W., Mitchell, D., Rème, H., Mazelle, C., Sauvaud, J. A., d'Uston, C., Cros, A., Medale, J. L., Bauer, S. J., Cloutier, P., Mayhew, M., Winterhalter, D., and Ness, N. F.: Magnetic Field and Plasma Observations at Mars: Initial Results of the Mars Global Surveyor Mission, Science, 279, 1676-1680, 1998.

Acuña, M. H., Connerney, J. E. P., Ness, N. F., Lin, R. P., Mitchell, D., Carlson, C. W., McFadden, J., Anderson, K. A., Rème, H., Mazelle, C., Vignes, D., Wasilewski, P., and Cloutier, P.: Global Distribution of Crustal Magnetization Discovered by the Mars Global Surveyor MAG/ER Experiment, Science, 284, 790-793, 1999.
Brain, D. A., Bagenal, F., Acuña, M. H., and Connerney, J. E. P.: Martian magnetic morphology: Contribution from the solar wind and crust, J. Geophys. Res., 108, 1424, doi:10.1029/2002JA009482, 2003.

Buehler, S. A., Eriksson, P., Kuhn, T., von Engeln, A., and Verdes, C.: ARTS, the atmospheric radiative transfer simulator, J. Quant. Spectrosc. Ra. 91, 65-93, doi:10.1016/j.jqsrt.2004.05.051, 2005.

Buehler, S. A., von Engeln, A., Brocard, E., John, V. O., Kuhn, T., and Eriksson, P.: Recent developments in the line-by-line modeling of outgoing longwave radiation, J. Quant. Spectrosc. Ra., 98, 446-457, doi:10.1016/j.jqsrt.2005.11.001, 2006.

Cain, J. C., Ferguson, B. B., and Mozzoni, D.: An $n=90$ internal potential function of the Martian crustal magnetic field, J. Geophys. Res., 108, 5008, doi:10.1029/2000JE001487, 2003.

Citron, R. I. and Zhong, S.: Constraints on the formation of the Martian crustal dichotomy from remnant crustal magnetism, Phys. Earth Planet. Int., 212-213, 55-63, 2012.

Connerney, J. E. P., Acuña, M. H., Ness, N. F., Spohn, T., and Schubert, G.: Mars Crustal Magnetism, Space Sci. Rev., 111, 1-32, 2004.

Davis, C. P., Evans, K. F., Buehler, S. A., Wu, D. L., and Pumphrey, H. C.: 3-D polarised simulations of space-borne passive $\mathrm{mm} / \mathrm{sub}$ mm midlatitude cirrus observations: a case study, Atmos. Chem. Phys., 7, 4149-4158, doi:10.5194/acp-7-4149-2007, 2007.

Eriksson, P., Jiménez, C., and Buehler, S. A.: Qpack, a general tool for instrument simulation and retrieval work, J. Quant. Spectrosc. Ra., 91, 47-64, doi:10.1016/j.jqsrt.2004.05.050, 2005.

Eriksson, P., Ekström, M., Melsheimer, C., and Buehler, S. A.: Efficient forward modelling by matrix representation of sensor responses, Int. J. Remote Sens., 27, 1793-1808, doi:10.1080/01431160500447254, 2006.

Eriksson, P., Buehler, S. A., Davis, C. P., Emde, C., and Lemke, O.: ARTS, the atmospheric radiative transfer simulator, Version 2, J. Quant. Spectrosc. Ra., 112, 1551-1558, 2011.

Forget, F., Hourdin, F., Foumier, R., Hourdin, C., and Talagran, O.: Improved general circulation models of the Martian atmosphere from the surface to above $80 \mathrm{~km}$, J. Geophys. Res., 104, 155-175, 1999.

Golubiatnikov, G. Y., Koshelev, M. A., and Krupnov, A. F.: Reinvestigation of pressure broadening parameters at $60-\mathrm{GHz}$ band and single $118.75 \mathrm{GHz}$ oxygen lines at room temperature, J. Mol. Spectrosc., 222, 191-197, 2003.

Hartogh, P., Lellouch, E., Crovisier, J., Banaszkiewicz, M., Bensch, F., Bergin, E., Billebaud, F., Biver, N., Blake, G., Błȩcka, M., Blommaert, J., Bockelee-Morvan, D., Cavalié, T., Cernicharo, J., Courtin, R., Davis, G., Decin, L., Encrenaz, P., Encrenaz, T., González, A., de Graauw, T., Hutsemekers, D., Jarchow, C., Jehin, E., Kidger, M., Küppers, M., de Lange, A., Lara, L.-M., Lis, D., Lorente, R., Manfroid, J., Medvedev, A., Moreno, R., Naylor, D., Orton, G., Portyankina, G., Rengel, M., Sagawa, H., Sánchez-Portal, M., Schieder, R., Sidher, S., Stam, D., Swinyard, B., Szutowicz, S., Thomas, N., Thornhill, G., Vandenbussche, B., Verdugo, E., Waelkens, C., and Walker, H.: Water and related chemistry in the solar system. A guaranteed time key programme for Herschel, Planet. Space Sci., 57, 1596-1606, doi:10.1016/j.pss.2009.07.009, 2009.

Hartogh, P., Błęcka, M., Jarchow, C., Sagawa, H., Lellouch, E., de Val-Borro, M., Rengel, M., Medvedev, A. S., Swinyard, B., Moreno, R., Cavalié, T., Lis, D., Banaszkiewicz, M., Bockelée- 
Morvan, D., Crovisier, J., Encrenaz, T., Küppers, M., Lara, L.M., Szutowicz, S., Vandenbussche, B., Bensch, F., Bergin, E. A., Billebaud, F., Biver, N., Blake, G., Blommaert, J., Cernicharo, J., Decin, L., Encrenaz, P., Feuchtgruber, H., Fulton, T., de Graauw, T., Jehin, E., Kidger, M., Lorente, R., Naylor, D., Portyankina, G., Sánchez-Portal, M., Schieder, R., Sidher, S., Thomas, N., Verdugo, E., Waelkens, C., Lorenzani, A., Tofani, G., Natale, E., Pearson, J., Klein, T., Leinz, C., Güsten, R., and Kramer, C.: First results on Martian carbon monoxide from Herschel/HIFI observations, Astron. Astrophys., 521, L48, doi:10.1051/00046361/201015159, 2010a.

Hartogh, P., Jarchow, C., Lellouch, E., Val-Borro, M., Rengel, M., Moreno, R., Medvedev, A., Sagawa, H., Swinyard, B., Cavalié, T., Lis, D., Błęcka, M., Banaszkiewicz, M., Bockelée-Morvan, D., Crovisier, J., Encrenaz, T., Küppers, M., Lara, L., Szutowicz, S., Vandenbussche, B., Bensch, F., Bergin, E. A., Billebaud, F., Biver, N., Blake, G., Blommaert, J., Cernicharo, J., Decin, L., Encrenaz, P., Feuchtgruber, H., Fulton, T., de Graauw, T., Jehin, E., Kidger, M., Lorente, R., Naylor, D., Portyankina, G., SánchezPortal, M., Schieder, R., Sidher, S., Thomas, N., Verdugo, E., Waelkens, C., Whyborn, N., Teyssier, D., Helmich, F., Roelfsema, P., Stutzki, J., LeDuc, H., and Stern, J.: Herschel/HIFI observations of Mars: first detection of $\mathrm{O}_{2}$ at submillimetre wavelengths and upper limits on $\mathrm{HCl}$ and $\mathrm{H}_{2} \mathrm{O}_{2}$, Astron. Astrophys., 521, L49, doi:10.1051/0004-6361/201015160, 2010b.

Ho, W., Birnbaum, G., and Rosenberg, A.: Far-Infrared CollisionInduced Absorption in $\mathrm{CO}_{2}$. I. Temperature Dependence, J. Chem. Phys., 55, 1028-1038, 1971.

Jakosky, B. M., Lin, R. P., Grebowsky, J. M., Luhmann, J. G., Mitchell, D. F., Beutelschies, G., Priser, T., Acuna, M., Andersson, L., Baird, D., Baker, D., Bartlett, R., Benna, M., Bougher, S., Brain, D., Carson, D., Cauffman, S., Chamberlin, P., Chaufray, J.-Y., Cheatom, O., Clarke, J., Connerney, J., Cravens, T., Curtis, D., Delory, G., Demcak, S., DeWolfe, A., Eparvier, F., Ergun, R., Eriksson, A., Espley, J., Fang, X., Folta, D., Fox, J., GomezRosa, C., Habenicht, S., Halekas, J., Holsclaw, G., Houghton, M., Howard, R., Jarosz, M., Jedrich, N., Johnson, M., Kasprzak, W., Kelley, M., King, T., Lankton, M., Larson, D., Leblanc, F., Lefevre, F., Lillis, R., Mahaffy, P., Mazelle, C., McClintock, W., McFadden, J., Mitchell, D. L., Montmessin, F., Morrissey, J., Peterson, W., Possel, W., Sauvaud, J.-A., Schneider, N., Sidney, W., Sparacino, S., Stewart, A. I. F., Tolson, R., Toublanc, D., Waters, C., Woods, T., Yelle, R., and Zurek, R.: The Mars Atmosphere and Volatile Evolution (MAVEN) Mission, Space Sci. Rev., 195, 3-48, doi:10.1007/s11214-015-0139-x, 2015.

Kasai, Y., Sagawa, H., Kuroda, T., Manabe, T., Ochiai, S., Kikuchi, K., Nishibori, T., Baron, P., Mendrok, J., Hartogh, P., Murtagh, D., Urban, J., von Schéele, F., and Frisk, U.: Overview of the Martian atmospheric submillimetre sounder FIRE, Planet. Space Sci., 63-64, 62-82, 2012.

Larsson, R., Ramstad, R., Mendrok, J., Buehler, S. A., and Kasai, Y.: A Method for Remote Sensing of Weak Planetary Magnetic Fields: Simulated Application to Mars, Geophys. Res. Lett., 40, 5014-5018, 2013.
Larsson, R., Buehler, S. A., Eriksson, P., and Mendrok, J.: A treatment of the Zeeman effect using Stokes formalism and its implementation in the Atmospheric Radiative Transfer Simulator (ARTS), J. Quant. Spectrosc. Ra., 133, 445-453, 2014.

Larsson, R., Milz, M., Rayer, P., Saunders, R., Bell, W., Booton, A., Buehler, S. A., Eriksson, P., and John, V. O.: Modeling the Zeeman effect in high-altitude SSMIS channels for numerical weather prediction profiles: comparing a fast model and a line-by-line model, Atmos. Meas. Tech., 9, 841-857, doi:10.5194/amt-9-841-2016, 2016.

Mendrok, J. and Eriksson, P.: Microwave Propagation Toolbox for Planetary Atmospheres - Final Report (D13a), Tech. rep., ESTEC Contract No 4000104175/11/NL/AF, 2014.

Morschauser, A., Lesur, V., and Grott, M.: A spherical harmonic model of the lithospheric magnetic fieldof Mars, J. Geophys. Res., 119, 1162-1188, 2014.

Navas-Guzmán, F., Kämpfer, N., Murk, A., Larsson, R., Buehler, S. A., and Eriksson, P.: Zeeman effect in atmospheric $\mathrm{O}_{2}$ measured by ground-based microwave radiometry, Atmos. Meas. Tech., 8 , 1863-1874, doi:10.5194/amt-8-1863-2015, 2015.

Nimmo, F. and Tanaka, K.: Early Crustal Evolution of Mars, Annu. Rev. Earth Planet. Sc., 33, 133-161, 2005.

Rodgers, C. D.: Inverse methods for atmospheric sounding: Theory and practice, Vol. 2, World Scientific Publishing Co. Pte. Ltd., 2000.

Sandel, B., Gröller, H., Yelle, R., Koskinen, T., Lewis, N., Bertaux, J.-L., Montmessin, F., and Quémerais, E.: Altitude profiles of $\mathrm{O}_{2}$ on Mars from SPICAM stellar occultations, Icarus, 252, 154160, doi:10.1016/j.icarus.2015.01.004, 2015.

Sobis, P., Drakinskiy, V., Wadefalk, N., Karandikhar, Y., Hammar, A., Emrich, A., Zhao, H., Bryllert, T., Tang, A.-Y., Nilsson, P.-A., Schleeh, J., Kim, H., Jacob, K., Murk, A., Grahn, J., and Stake, J.: Low noise GaAs Schottky TMIC and InP Hemt MMIC based receivers for the ISMAR and SWI instruments, Tech. rep., ESAESTEC, Noordwijk, the Netherlands, 2014.

Sobis, P. J., Emrich, A., and Stake, J.: A Low VSWR 2SB Schottky Receiver, IEEE Transactions on Terahertz Science and Technology, 1, 403-411, doi:10.1109/TTHZ.2011.2166176, 2011.

Treuttel, J., Gatilova, L., Maestrini, A., Moro-Melgar, D., Yang, F., Tamazouzt, F., Vacelet, T., Jin, Y., Cavanna, A., Matéos, J., Féret, A., Chaumont, C., and Goldstein, C.: A 520-620-GHz Schottky Receiver Front-End for Planetary Science and Remote Sensing With 1070 K-1500 K DSB Noise Temperature at Room Temperature, IEEE Transactions on Terahertz Science and Technology, 6, 148-155, doi:10.1109/TTHZ.2015.2496421, 2016.

Zeeman, P.: On the Influence of Magnetism on the Nature of the Light Emitted by a Substance, Astrophys. J., 5, 332-347, doi:10.1086/140355, 1897. 